\title{
Utility-optimal Cross-layer Design for WLAN with MIMO Channels
}

\author{
Yuxia Lin and Vincent W.S. Wong \\ Department of Electrical and Computer Engineering \\ The University of British Columbia, Vancouver, BC, Canada, V6T 1Z4 \\ Email: \{yuxial, vincentw\}@ece.ubc.ca
}

\begin{abstract}
Wireless local area networks (WLANs) have become a ubiquitous high-speed data access technology. The recent 802.11n proposal further increases the transmission rate by using the multiple-input-multiple-output (MIMO) technique. Multiple antennas can be used to achieve a performance gain by either increasing the transmission reliability through spatial diversity or increasing the transmission rate through spatial multiplexing. This new characteristic at the wireless physical layer requires the corresponding adaptation at the medium access control (MAC) layer to reach the best performance gain. This paper proposes a cross-layer optimization framework for jointly optimizing the MIMO configuration at the physical layer and the persistent probabilities for different classes of multimedia traffic at the MAC layer with slotted Aloha. A distributed algorithm NUM$D$ based on dual decomposition is proposed. Numerical results are compared with a simplified algorithm $N U M-S$ to show the effectiveness of the proposed methods.
\end{abstract}

\section{INTRODUCTION}

The IEEE 802.11-based wireless local area networks (WLANs) have been widely recognized as one of the most successful technologies for providing high-speed wireless data access for hotspot locations. The current $802.11 \mathrm{a} / \mathrm{g}$ standards provide a raw data rate of up to 54 Mbps. By utilizing the multiple-input-multiple-output (MIMO) technology at the physical layer, the recent $802.11 \mathrm{n}$ proposal [1] aims to increase the physical link data rate up to $600 \mathrm{Mbps}$.

There are two major performance gains in a wireless MIMO system: the diversity gain and the spatial multiplexing gain [2]. In a fading channel, multiple antennas can increase the system reliability through spatial diversity. Each pair of the transmit and receive antennas provides one signal path from the transmitter to the receiver. If the same information symbol is transmitted across different paths and each path experiences fading independently, then multiple independently faded data symbols can be received at the receiver. Hence, the reliability of the transmission is enhanced. On the other hand, if we transmit independent data symbols on each transmit antenna, spatial multiplexing gain can be achieved, which significantly increases the spectral efficiency.

Let $\gamma$ denote the average signal-to-noise-ratio (SNR) of a wireless link. A wireless MIMO system is said to achieve spatial multiplexing gain $r$ and diversity gain $d$ if the data rate $R(\gamma)$ and the average error probability $P_{e}(\gamma)$ have these asymptotic characteristics in the high SNR regime [3]:

$$
\lim _{\gamma \rightarrow \infty} \frac{R(\gamma)}{\log \gamma}=r, \quad \text { and } \quad \lim _{\gamma \rightarrow \infty} \frac{\log P_{e}(\gamma)}{\log \gamma}=-d
$$

For a point-to-point wireless link with $M_{T}$ transmit antennas and $M_{R}$ receive antennas, the maximal (full) diversity gain is $M_{T} M_{R}$. The maximal spatial multiplexing gain is $\min \left\{M_{T}, M_{R}\right\}$. However, it is not possible to realize both the full diversity gain and the maximal spatial multiplexing gain at the same time. There is a fundamental tradeoff between these two performance gains. For each spatial multiplexing gain $r$, the best diversity gain (denoted as $d^{*}(r)$ ) is the supremum of the diversity gain achieved over all schemes. In a Rayleigh-fading channel with long enough block lengths, the optimal multiplexing-diversity tradeoff $d^{*}(r)$ is given by the piecewise-linear function connecting the points $\left(r, d^{*}(r)\right)$, for $r=0,1, \ldots, \min \left\{M_{T}, M_{R}\right\}$ [3]:

$$
d^{*}(r)=\left(M_{T}-r\right)\left(M_{R}-r\right) .
$$

In this paper, we assume that a family of carefully designed codes can achieve the above optimal tradeoff performance.

In the research literature, some cross-layer designs only focus on utilizing one of the two MIMO resources: either diversity or spatial multiplexing. For example, a MIMOenabled MAC protocol was proposed in [4], where the protocol mitigates interferences by utilizing the multiplexing capability of the MIMO system. Other schemes allow the system to adaptively switch between the two MIMO working modes. The adaptive routing protocol in [5] directs a link to operate under full spatial diversity gain when the distance is long and the signal is weak. It operates under maximal spatial multiplexing gain when the distance is short and the signal is strong so as to increase throughput. The transmission control protocol (TCP) over wireless MIMO channel is shown to perform better with a more reliable link provided by the diversity gain of a spacetime block coding system at low SNR regions, while a spatial multiplexing scheme outperforms in the high SNR regions [6].

As shown in (2), apart from the two working modes where we obtain a maximal multiplexing gain $r$ while the diversity gain $d$ is zero (or vice versa), there are intermediate working modes where we can achieve part of the diversity gain and multiplexing gain simultaneously. It is intuitive to consider whether adaptively tuning the MIMO system gains along the optimal trade-off curve provided by (2) is more beneficial than only choosing the points on the two ends of this curve.

In [7], Lee et al. studied the adaptive channel coding in a wireless ad-hoc network by formulating the rate-reliability tradeoff problem as a network utility maximization (NUM) 
problem. The NUM architecture has been shown to be an effective way of tackling cross-layer optimization problems in wireless networks [8]. In [9], algorithms are proposed to jointly select the MIMO coding scheme and the contention window size at the MAC layer.

In this paper, we propose a NUM framework for WLAN with MIMO antennas. Multiple access categories' traffic is being considered. Instead of operating the MIMO system in either full-diversity or full-multiplexing mode, we investigate the optimal multiplexing-diversity gain tradeoff to achieve the best network utility. The NUM framework naturally leads us to a cross-layer design architecture where the MIMO multiplexing-diversity tradeoff at the physical layer is coupled with tuning the persistent probabilities at the MAC layer.

The contributions of our work are as follows:

1) We propose a cross-layer design framework called $N U M-O$ which jointly selects the MIMO coding scheme at the physical layer and the transmission persistent probability at the MAC layer to achieve the maximal network utility.

2) A distributed solution $N U M-D$ is proposed for the $N U M$ $O$ framework by using the dual decomposition method.

3) We also propose another scheme called NUM-S which is simpler and practical for implementation and is used as a baseline for performance comparison.

4) We present numerical results to show the effectiveness of the proposed $N U M-D$ scheme and compare it with $N U M-S$.

The rest of the paper is organized as follows. The system model is presented in Section II. Section III proposes the utility-based cross-layer design framework $N U M-O$, and the distributed solution $N U M-D$. A simplified scheme $N U M-S$ is proposed in Section IV to be used as a baseline for performance analysis. Section V presents numerical results of the proposed schemes. Conclusions and future work are given in Section VI.

\section{SYSTEM MODEL}

Consider a WLAN with one access point (AP). We assume that there are two access categories (ACs) of traffic in the network. Let $\mathcal{N}_{1}$ denote the set of wireless stations sending best-effort traffic (AC_BE), and $\mathcal{N}_{2}$ denote the set of stations sending real-time traffic (AC_RT), which has higher priority. Each station transmits one class of traffic to the AP. As a result, $\mathcal{N}=\mathcal{N}_{1} \cup \mathcal{N}_{2}$ represents the set of all the nodes in the network. We use $N_{1}, N_{2}$, and $N$ to denote the number of stations in the set. Thus, $N_{1}=\left|\mathcal{N}_{1}\right|, N_{2}=\left|\mathcal{N}_{2}\right|$, and $N=|\mathcal{N}|$, where $|$.$| denotes the cardinality of a set. The real-$ time traffic are assumed to be constant-bit-rate (CBR) flows. Extension to four ACs is fairly straightforward based on the optimization framework proposed below.

Each station is assumed to have $M_{T}$ transmit antennas. The AP is assumed to have $M_{R}$ receive antennas. Each wireless link can utilize some multi-antenna coding scheme to achieve the optimal diversity-multiplexing tradeoff given by (2). Since the curve in (2) is non-differentiable, we use the differentiable approximation as in [7]:

$$
d(r)=\left(M_{T}-r\right)\left(M_{R}-r\right), 0 \leq r \leq \min \left\{M_{T}, M_{R}\right\}
$$

If a wireless link from station $s \in \mathcal{N}$ to the AP has SNR $\gamma_{s}$, multiplexing gain $r_{s}$ and diversity gain $d_{s}$, then the link data rate $c_{s}\left(\gamma_{s}\right)$ (bps) and error probability $p_{s}^{e r r}\left(\gamma_{s}\right)$ can be approximated as [7]:

$$
\begin{array}{ll}
c_{s}\left(\gamma_{s}\right)=k_{c} r_{s} \log \gamma_{s}, & s \in \mathcal{N}, \\
p_{s}^{e r r}\left(\gamma_{s}\right)=k_{p} \gamma_{s}^{-d\left(r_{s}\right)}, & s \in \mathcal{N}
\end{array}
$$

where $k_{c}$ and $k_{p}$ are positive constants for different coding schemes. The log function uses base 2 throughout this paper. The multiplexing gain $r_{s}$ and diversity gain $d_{s}$ conform to the optimal tradeoff in (3).

When source $s$ transmits with persistent probability $p_{s}$ by slotted Aloha, the data rate is:

$$
x_{s}=c_{s}\left(\gamma_{s}\right) p_{s} \prod_{k \in \mathcal{N}, k \neq s}\left(1-p_{k}\right), \quad s \in \mathcal{N} .
$$

From the channel error probability in (5), the link transmission reliability $y_{s}$ is:

$$
y_{s}=1-k_{p} \gamma_{s}^{-d\left(r_{s}\right)}, \quad s \in \mathcal{N} .
$$

There are some constraints on the above source rates and link reliability. The $N_{2}$ real-time CBR traffic has a hard throughput requirement $R$ and reliability demand $Q$ :

$$
\begin{array}{ll}
R \leq x_{j} \leq \hat{x}=k_{c} \hat{r} \log \hat{\gamma}, & j \in \mathcal{N}_{2}, \\
Q \leq y_{j} \leq 1, & j \in \mathcal{N}_{2} .
\end{array}
$$

where $\hat{x}$ is the constant upper bound on throughput, with $\hat{r}$ and $\hat{\gamma}$ being the network's maximum multiplexing gain and maximum SNR value, respectively.

The AC_BE traffic also has the bounding constraints:

$$
\begin{array}{ll}
\check{x}_{1} \leq x_{i} \leq \hat{x}, & i \in \mathcal{N}_{1}, \\
\check{y}_{1} \leq y_{i} \leq 1, & i \in \mathcal{N}_{1} .
\end{array}
$$

where $\check{x}_{1}$ and $\check{y}_{1}$ are the constant lower bound on throughput and reliability for best-effort traffic, respectively.

There is an upper bound on the spatial multiplexing gain:

$$
0 \leq r_{s} \leq \min \left\{M_{T}, M_{R}\right\}, \quad s \in \mathcal{N} .
$$

We next propose the appropriate network utility functions to measure the network QoS performance.

\section{A. Network Utility Formulation}

The utility function for each source node in a NUM framework is usually chosen such that it reflects the satisfaction attained by the user for the services that it receives. It can also be interpreted as the revenue that the network operator would be able to accrue by providing a certain level of QoS service to the end users. A commonly used family of utility functions is [10]:

$$
U(x)= \begin{cases}(1-\alpha)^{-1} x^{(1-\alpha)}, & \text { if } \alpha \neq 1, \\ \log x, & \text { if } \alpha=1\end{cases}
$$


where $\alpha$ is the fairness parameter. For example, if $x$ is the source node's throughput, then $\alpha=0$ leads to throughput maximization. Proportional and harmonic mean fairness are achieved when $\alpha=1$ and $\alpha=2$, respectively. When $\alpha \rightarrow \infty$, max-min fairness can be achieved.

In our NUM framework, we choose the utility function for the wireless station with AC_BE traffic as:

$$
U_{1}\left(x_{i}, y_{i}\right)=\frac{\left(x_{i} y_{i}\right)^{1-\alpha}-\left(\check{x}_{1} \check{y}_{1}\right)^{1-\alpha}}{(\hat{x})^{1-\alpha}-\left(\check{x}_{1} \check{y}_{1}\right)^{1-\alpha}}, \quad \alpha>1
$$

where the product $x_{i} y_{i}$ can be interpreted as the effective throughput for the best-effort station $i \in \mathcal{N}_{1}$.

For the AC_RT's CBR traffic, the utility function is chosen to be a function of the reliability because we expect that the data rate $x$ is at least equal to the CBR's constant data rate:

$$
U_{2}\left(y_{i}\right)=\frac{y_{i}^{1-\alpha}-Q^{1-\alpha}}{1-Q^{1-\alpha}}, \quad \alpha>1 .
$$

The utility functions in (12) and (13) are normalized such that $U_{1}\left(\check{x}_{1}, \check{y}_{1}\right)=U_{2}(Q)=0$ and $U_{1}(\hat{x}, 1)=U_{2}(1)=1$. This avoids the apparent problem of combining two utility functions which would otherwise have a few magnitude of difference in value. The network utility is the sum of the utilities of all the source nodes:

$$
U(\mathbf{x}, \mathbf{y})=\sum_{i \in \mathcal{N}_{1}} \beta U_{1}\left(x_{i}, y_{i}\right)+\sum_{j \in \mathcal{N}_{2}}(1-\beta) U_{2}\left(y_{j}\right), \alpha>1
$$

where $\mathbf{x}, \mathbf{y}$ are vectors for each source's throughput and reliability, $\beta$ is an adjustable parameter between 0 and 1 to tune the weight of AC_BE and AC_RT's utilities on the network utility function. It can be shown that $U(\mathbf{x}, \mathbf{y})$ is a concave function.

\section{Utility-Optimal PHY/MAC CROSS-LAYER DESIGN}

\section{A. Centralized Scheme: NUM-O}

We now present our first design, which is called NUM$O$. Assuming that each station $s$ can adaptively adjust its persistent probability $p_{s}$ at the MAC layer with cross-layer information from the MIMO-enabled physical layer, we propose the following utility maximization formulation:

$$
\begin{aligned}
& \max _{\mathbf{x}, \mathbf{y}} \sum_{i \in \mathcal{N}_{1}} \beta U_{1}\left(x_{i}, y_{i}\right)+\sum_{j \in \mathcal{N}_{2}}(1-\beta) U_{2}\left(y_{j}\right) \\
& \text { s.t. } \quad x_{s} \leq k_{c} r_{s} \log \left(\gamma_{s}\right) p_{s} \prod_{k \in \mathcal{N}, k \neq s}\left(1-p_{k}\right), \quad \forall s \in \mathcal{N} \\
& y_{s} \leq 1-k_{p} \gamma_{s}^{-\left(M_{T}-r_{s}\right)\left(M_{R}-r_{s}\right)}, \forall s \in \mathcal{N} \\
& \check{x}_{1} \leq x_{i} \leq \hat{x}, \quad \forall i \in \mathcal{N}_{1} \\
& R \leq x_{j} \leq \hat{x}, \quad \forall j \in \mathcal{N}_{2} \\
& \check{y}_{1} \leq y_{i} \leq 1, \quad \forall i \in \mathcal{N}_{1} \\
& Q \leq y_{j} \leq 1, \quad \forall j \in \mathcal{N}_{2} \\
& 0 \leq r_{s} \leq \min \left\{M_{T}, M_{R}\right\}, \quad \forall s \in \mathcal{N} \\
& 0 \leq p_{s} \leq 1, \quad \forall s \in \mathcal{N} \text {. }
\end{aligned}
$$

Although problem (15) is a non-linear optimization problem with respect to the variables $\{\mathbf{x}, \mathbf{y}, \mathbf{r}, \mathbf{p}\}$, it can be transformed to a convex optimization problem by a change of variables and placing an upper bound on $\mathbf{r}$. We choose $\alpha>1$ and perform a $\log$ transformation of variables: $x_{s}^{\prime}=\log x_{s}, p_{s}^{\prime}=\log p_{s}$ and $p_{s}^{\prime \prime}=\log \left(1-p_{s}\right)$ for $s \in \mathcal{N}$. The NUM problem becomes:

$$
\begin{aligned}
& \max _{\mathbf{x}^{\prime}, \mathbf{y}} \sum_{i \in \mathcal{N}_{1}} \beta U_{1}^{\prime}\left(x_{i}^{\prime}, y_{i}\right)+\sum_{j \in \mathcal{N}_{2}}(1-\beta) U_{2}\left(y_{j}\right) \\
& \text { s.t. } \quad x_{s}^{\prime}-\log k_{c}-\log r_{s}-\log \left(\log \gamma_{s}\right)-p_{s}^{\prime} \\
& -\sum_{k \in \mathcal{N}, k \neq s} p_{k}^{\prime \prime} \leq 0, \quad \forall s \in \mathcal{N} \\
& y_{s}-1+k_{p} \gamma_{s}^{-\left(M_{T}-r_{s}\right)\left(M_{R}-r_{s}\right)} \\
& \leq 0 \text {, } \\
& \forall s \in \mathcal{N} \\
& e^{p_{s}^{\prime}}+e^{p_{s}^{\prime \prime}} \leq 1 \\
& \forall s \in \mathcal{N} \\
& \log \check{x}_{1} \leq x_{i}^{\prime} \leq \log \hat{x}, \\
& \forall i \in \mathcal{N}_{1} \\
& \log R \leq x_{j}^{\prime} \leq \log \hat{x}, \\
& \forall j \in \mathcal{N}_{2} \\
& \check{y}_{1} \leq y_{i} \leq 1 \text {, } \\
& \forall i \in \mathcal{N}_{1} \\
& Q \leq y_{j} \leq 1 \text {, } \\
& \forall j \in \mathcal{N}_{2} \\
& 0 \leq r_{s} \leq \Delta_{s}, \quad p_{s}^{\prime}, p_{s}^{\prime \prime} \leq 0, \quad \forall s \in \mathcal{N}
\end{aligned}
$$

where

$$
\begin{gathered}
\Delta_{s}=\min \left\{\min \left\{M_{T}, M_{R}\right\}, \frac{M_{T}+M_{R}}{2}-\frac{1}{2} \sqrt{\frac{2}{\ln \gamma_{s}}}\right\}, \\
U_{1}^{\prime}\left(x^{\prime}, y\right)=\frac{\left(e^{x^{\prime}} y\right)^{1-\alpha}-\left(\check{x}_{1} \check{y}_{1}\right)^{1-\alpha}}{(\hat{x})^{1-\alpha}-\left(\check{x}_{1} \check{y}_{1}\right)^{1-\alpha}}
\end{gathered}
$$

Now the above problem is a convex optimization problem. It can be solved by using commercial software package (e.g., CPLEX). In a WLAN where all the wireless stations communicate with the AP directly, the AP can measure each link's SNR $\left(\gamma_{s}\right)$ and use this information to solve for the persistent probability $p_{s}$ at the MAC layer, and the multiplexing gain $r_{s}$ at the MIMO physical layer. The resulting $r_{s}$ and $p_{s}$ values can be sent to each wireless station via piggyback on the control frames. Each station will then tune its operating parameters accordingly. Furthermore, the AP can adjust the parameter $\beta$ in the network utility function to adaptively tune the tradeoff between enhancing best-effort traffic's throughput and realtime flow's reliability.

\section{B. Distributed Scheme: NUM-D}

The NUM-O problem proposed in (15) is a centralized scheme, where the AP acts as the central controller and distributes the control information to each wireless station. This may suffer from a single-point failure. A more attractive scheme would be a fully distributed algorithm, where each wireless station can decide its own operation parameter based on locally available information and limited message communications with the AP. Since problem (16) is convex, the dual decomposition approach can be used to obtain the distributed solution. By relaxing the first two constraints, the Lagrangian 
function for problem (16) is:

$$
\begin{aligned}
& L\left(\mathbf{x}^{\prime}, \mathbf{y}, \mathbf{r}, \mathbf{p}^{\prime}, \mathbf{p}^{\prime \prime}, \boldsymbol{\lambda}, \boldsymbol{\mu}\right) \\
= & \sum_{i \in \mathcal{N}_{1}} \beta U_{1}^{\prime}\left(x_{i}^{\prime}, y_{i}\right)+\sum_{j \in \mathcal{N}_{2}}(1-\beta) U_{2}\left(y_{j}\right) \\
& +\sum_{s \in \mathcal{N}} \lambda_{s}\left[\log k_{c}+\log r_{s}+\log \left(\log \gamma_{s}\right)+p_{s}^{\prime}+\sum_{k \neq s} p_{k}^{\prime \prime}\right. \\
& \left.-x_{s}^{\prime}\right]+\sum_{s \in \mathcal{N}} \mu_{s}\left[1-y_{s}-k_{p} \gamma_{s}^{-\left(M_{T}-r_{s}\right)\left(M_{R}-r_{s}\right)}\right]
\end{aligned}
$$

The Lagrange dual function is:

$$
\Phi(\boldsymbol{\lambda}, \boldsymbol{\mu})=\max _{\mathbf{x}^{\prime}, \mathbf{y}, \mathbf{r}, \mathbf{p}^{\prime}, \mathbf{p}^{\prime \prime}} L\left(\mathbf{x}^{\prime}, \mathbf{y}, \mathbf{r}, \mathbf{p}^{\prime}, \mathbf{p}^{\prime \prime}, \boldsymbol{\lambda}, \boldsymbol{\mu}\right)
$$

where $\mathbf{x}^{\prime}, \mathbf{y}, \mathbf{r}, \mathbf{p}^{\prime}, \mathbf{p}^{\prime \prime}$ are subject to the third to the ninth constraints of (16).

The dual optimization problem can be formulated as:

$$
\begin{array}{ll}
\min & \Phi(\boldsymbol{\lambda}, \boldsymbol{\mu}) \\
\text { s.t. } & \boldsymbol{\lambda} \succeq \mathbf{0}, \text { and } \boldsymbol{\mu} \succeq \mathbf{0} .
\end{array}
$$

We can further consider decomposing the problem into two sub-problems. The maximization of the Lagrangian over $\mathbf{x}^{\prime}, \mathbf{y}, \mathbf{r}, \mathbf{p}^{\prime}, \mathbf{p}^{\prime \prime}$ can be conducted in parallel at the application layer for the transmission rate $\mathbf{x}^{\prime}$ and reliability $\mathbf{y}$ :

$$
\begin{array}{lll}
\max & \sum_{i \in \mathcal{N}_{1}} \beta U_{1}^{\prime}\left(x_{i}^{\prime}, y_{i}\right)+\sum_{j \in \mathcal{N}_{2}}(1-\beta) U_{2}\left(y_{j}\right) \\
& -\sum_{s \in \mathcal{N}}\left[\lambda_{s} x_{s}^{\prime}+\mu_{s} y_{s}\right] & \\
\text { s.t. } & \log \check{x}_{1} \leq x_{i}^{\prime} \leq \log \hat{x}, & \forall i \in \mathcal{N}_{1} \\
& \log R \leq x_{j}^{\prime} \leq \log \hat{x}, & \forall j \in \mathcal{N}_{2} \\
& \check{y}_{1} \leq y_{i} \leq 1, & \forall i \in \mathcal{N}_{1} \\
& Q \leq y_{j} \leq 1, & \forall j \in \mathcal{N}_{2}
\end{array}
$$

And, on the MAC/PHY layers for the transmission probability $\mathbf{p}$ and MIMO diversity gain $\mathbf{r}$ :

$$
\begin{array}{lll}
\max & \sum_{s \in \mathcal{N}}\left[\lambda_{s}\left(\log r_{s}+p_{s}^{\prime}+\sum_{k \neq s} p_{k}^{\prime \prime}\right)\right. & \\
& \left.-\mu_{s} k_{p} \gamma_{s}^{-\left(M_{T}-r_{s}\right)\left(M_{R}-r_{s}\right)}\right] & \\
\text { s.t. } & e^{p_{s}^{\prime}}+e^{p_{s}^{\prime \prime}} \leq 1, & \forall s \in \mathcal{N} \\
& p_{s}^{\prime}, p_{s}^{\prime \prime} \leq 0, & \forall s \in \mathcal{N} \\
& 0 \leq r_{s} \leq \Delta_{s}, & \forall s \in \mathcal{N} .
\end{array}
$$

The above problem can be separately solved at each wireless station locally. For $i \in \mathcal{N}_{1}$ with AC_BE traffic, it solves the following problem to obtain its $x_{i}^{\prime}$ and $y_{i}$ values:

$$
\begin{array}{cl}
\max & \beta U_{1}^{\prime}\left(x_{i}^{\prime}, y_{i}\right)-\left(\lambda_{i} x_{i}^{\prime}+\mu_{i} y_{i}\right) \\
\text { s. t. } & \log \check{x}_{1} \leq x_{i}^{\prime} \leq \log \hat{x}, \\
& \check{y}_{1} \leq y_{i} \leq 1 .
\end{array}
$$

For each wireless station $j \in \mathcal{N}_{2}$ with AC_RT traffic, its optimal $x_{j}^{\prime}$ and $y_{j}$ values can be calculated from:

$$
\begin{array}{cl}
\max & (1-\beta) U_{2}\left(y_{j}\right)-\left(\lambda_{j} x_{j}^{\prime}+\mu_{j} y_{j}\right) \\
\text { s.t. } & \log R \leq x_{j}^{\prime} \leq \log \hat{x}, \\
& Q \leq y_{i} \leq 1 .
\end{array}
$$

For each wireless station $s \in N$, the MIMO diversity gain $\mathbf{r}$ at the PHY layer can be calculated from:

$$
\begin{array}{ll}
\max & \lambda_{s} \log r_{s}-\mu_{s} k_{p} \gamma_{s}^{-\left(M_{T}-r_{s}\right)\left(M_{R}-r_{s}\right)} \\
\text { s.t. } & 0 \leq r_{s} \leq \Delta_{s} .
\end{array}
$$

The transmission probability $\mathbf{p}$ can be solved at each wireless station by:

$$
\begin{array}{ll}
\max & \lambda_{s} p_{s}^{\prime}+\sum_{k \in \mathcal{N}, k \neq s} \lambda_{k} p_{s}^{\prime \prime} \\
\text { s.t. } & e^{p_{s}^{\prime}}+e^{p_{s}^{\prime \prime}} \leq 1, \\
& p_{s}^{\prime}, p_{s}^{\prime \prime} \leq 0 .
\end{array}
$$

With the subgradient projection algorithm, $\lambda_{s}$ and $\mu_{s}$ can be updated at each station as follows:

$$
\begin{aligned}
& \lambda_{s}(t+1)= {\left[\lambda_{s}(t)-\delta(t)\left(\log r_{s}+p_{s}^{\prime}+\sum_{k \neq s} p_{k}^{\prime \prime}+\log k_{c}\right.\right.} \\
&\left.\left.+\log \log \gamma_{s}-x_{s}^{\prime}\right)\right]^{+}, \\
& \mu_{s}(t+1)=\left[\mu_{s}(t)-\delta(t)\left(1-y_{s}-k_{p} \gamma_{s}^{-\left(M_{T}-r_{s}\right)\left(M_{R}-r_{s}\right)}\right]^{+}\right.
\end{aligned}
$$

where $[x]^{+}=\max \{x, 0\}$, and $\delta(t)$ is the diminishing step size [11] (e.g., $\delta(t)=1 / 1+t$ ). By taking the advantage of the broadcast nature of the wireless transmission, the values of $\boldsymbol{\lambda}$ and $\boldsymbol{p}$ which are required in each iteration can be piggybacked over a broadcast frame by each station. Thus, it introduces limited overhead in the network. The distributed algorithm (24) to (29) runs at each wireless station $s$ by calculating its target throughput $x_{s}$, reliability requirement $y_{s}$, MIMO diversity gain $r_{s}$, and transmission probability $p_{s}$. The $r_{s}$ and $p_{s}$ results can then be used to adjust the PHY/MAC operation parameters.

\section{NUM-S: UTILITY-MAXIMIZATION WITH SEPARATED PHY/MAC LAYERS}

For performance analysis, we propose a simplified scheme in which the persistent probabilities at the MAC layer and the multiplexing-diversity tradeoff scheme at the physical layer are determined separately. We call this scheme $N U M-S$, and use it as a baseline for performance comparison in Section V.

At the MAC layer, it is shown that under identical traffic and data rate, the network throughput is maximized with each node transmitting with persistent probability [12]:

$$
p_{s}=1 / N, \quad s \in \mathcal{N} \text {. }
$$

where $N$ is used interchangeably to denote both the set and the total number of wireless stations. If we choose the persistent probability based on (30), then the MIMO multiplexingdiversity tradeoff at the physical layer for each station becomes independent of each other and can be computed in the following steps: At the physical layer, the AC_RT station $j$ can calculate its required level of MIMO multiplexing gain for providing the CBR data rate $R$ from (6):

$$
r_{j}=\frac{R N^{N}}{k_{c} \log \gamma_{j}(N-1)^{N-1}}, \quad j \in \mathcal{N}_{2} .
$$


This $r_{j}$ is the minimum multiplexing gain required for sustaining the data rate $R$, which leads to the corresponding maximum diversity gain from (3) because $d_{j}$ is a strictly decreasing function of $r_{j}$. This maximum diversity gain $d_{j}$ in turn provides the maximum reliability $y_{j}$ available for station $j$ as from (7). Because the utility function (13) is strictly increasing with $y_{j}$, this multiplexing-diversity tradeoff maximizes the utility for AC_RT stations.

For the AC_BE stations, maximizing $U_{B E}(x y)$ in (18) is equivalent to maximizing $x y$ because it is strictly increasing with $x y$. We can solve for the optimal multiplexing gain $r_{i}$ for each AC_BE station $i \in N_{1}$ with the following simple constrained maximization problem:

$$
\begin{array}{cl}
\max _{r_{i}} & k_{c} r_{i} \log \left(\gamma_{i}\right) \frac{(N-1)^{N-1}}{N^{N}}\left(1-k_{p} \gamma_{i}^{-d\left(r_{i}\right)}\right) \\
\text { subject to } & \check{x}_{1} \leq k_{c} r_{i} \log \left(\gamma_{i}\right) \frac{(N-1)^{N-1}}{N^{N}} \leq \hat{x} \\
& \check{y}_{1} \leq 1-k_{p} \gamma_{s}^{-d\left(r_{i}\right)} \leq 1 \\
& d\left(r_{i}\right)=\left(M_{T}-r_{i}\right)\left(M_{R}-r_{i}\right) \\
& 0 \leq r_{i} \leq \min \left\{M_{T}, M_{R}\right\}
\end{array}
$$

The solution of problem (32) provides each AC_BE station the multiplexing gain $r_{i}$ to be used on its MIMO transmission scheme. The resulting data rate $x_{i}$ and transmission reliability $y_{i}$ can be subsequently calculated from (6) and (7). With AC_RT stations' reliability results, we can obtain the network utility from (16).

\section{NUMERICAL RESULTS}

We study the performance of $N U M-D$ and $N U M-S$ with numerical tests in Matlab. The following parameters are used: $k_{c}=20 \mathrm{MHz}, k_{p}=0.15, \alpha=1.1, M_{T}=2, M_{R}=3$, $\check{x}=0.01 \mathrm{Mbps}, \hat{\gamma}=30 \mathrm{~dB}, \check{y}_{1}=0.7$, and $Q=0.85$. The stopping criteria for $N U M-D$ is that $\left|\lambda_{s}(t+1)-\lambda_{s}(t)\right|$ and $\left|\mu_{s}(t+1)-\mu_{s}(t)\right|$ are less than $10^{-6}$ for all $s$. Each station's SNR value $\gamma_{s}$ is a function of the transmit power and wireless channel models. For computation simplicity, we assume that $\gamma_{s}$ is normally distributed with parameters $\left[\gamma_{m}, \gamma_{v}\right]$, where $\gamma_{m}$ and $\gamma_{v}$ are the mean and variance of the normal distribution. This is an approximation for a WLAN where each wireless node is stationed in similar distances to the AP. Thus, their SNRs are closely distributed around a mean value $\gamma_{m}$. Other assumptions such as uniformly distributed SNRs have also been tested, and do not seem to have an impact on the performance comparisons carried out below.

\section{A. Effect of $\beta$ on the Network Utility Function}

First, we examine the effectiveness of the network utility function (14) in determining the tradeoff between AC_BE's throughput and AC_RT's reliability. We vary the parameter $\beta$ from 0.6 to 1 with a step size of 0.05 , and solve problem (16). The results for $N_{1}=N_{2}=5, \gamma_{m}=8 d B, \gamma_{v}=0.5 \gamma_{m}$, and varying $R$ are shown in Figure 1 . We can see that for a smaller $\beta$, the AC_BE's throughput is given lower priority, but the network achieves higher reliability for the AC_RT traffic. As

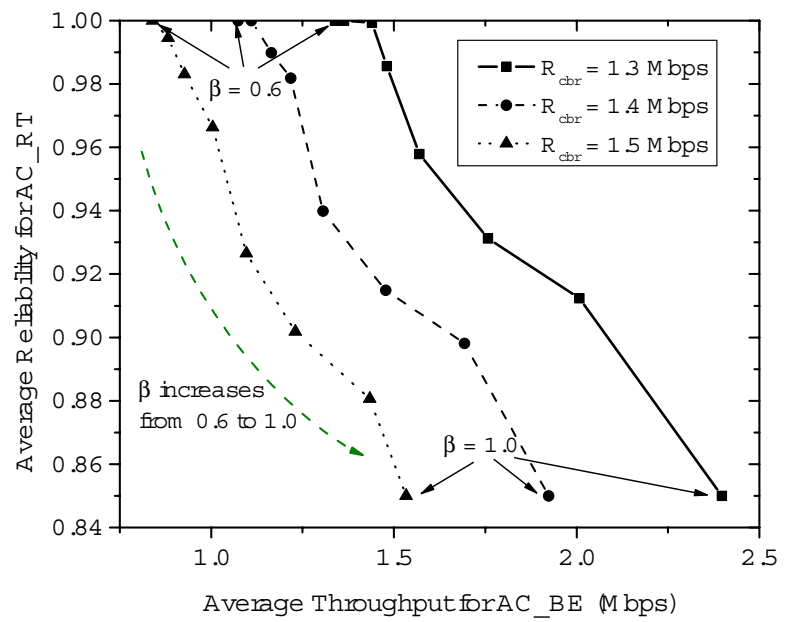

Fig. 1. The tradeoff between throughput and reliability.

$\beta$ increases, the AC_BE's throughput significantly increases at the price of decreasing reliability on AC_RT traffic. The area below each curve is effectively the achievable throughputreliability region for a specified $R$. When $R$ increases, this region shrinks because more resources have to be allocated to guarantee the higher throughput requirement from the AC_RT traffic.

Figure 1 shows that the utility function (14) has great flexibility in adapting different user QoS requirements by adjusting the parameter $\beta$. If the network operators can generate higher revenues by providing high reliability for AC_RT traffic, they may tend to choose a smaller $\beta$ value. In the following tests, we choose $\beta=0.85$.

\section{B. Performance under Different Number of Stations}

In this experiment, $\gamma_{m}$ is fixed to be $8 \mathrm{~dB}$, and $\gamma_{v}=0.5 \gamma_{m}$. We vary the number of stations in each AC from 1 to $5 . R$ is set at $1.5 \mathrm{Mbps}$. The resulting network utility, AC_BE's throughput and AC_RT's reliability performance are shown in Figure 2. When the number of stations is small, the NUM-D scheme achieves much higher AC_BE throughput than NUM-S. When the number of stations increases, the performance gain on AC_BE's throughput by NUM-D diminishes. But NUM$D$ is able to maintain the AC_RT's reliability above $95 \%$, while $N U M-S$ 's reliability performance significantly deteriorates. Overall, the network utility achieved by $N U M-D$ is consistently higher than $N U M-S$.

\section{Performance under Different SNRs}

In this experiment, $N_{1}$ and $N_{2}$ are fixed at 5. The network average $\gamma_{m}$ varies from $6 \mathrm{~dB}$ to $16 \mathrm{~dB}, \gamma_{v}=0.5 \gamma_{m}$. $R$ is set at $1.0 \mathrm{Mbps}$. Figure 3 shows the network utility, AC_BE's throughput and AC_RT's reliability achieved with these two schemes. Results show that in the lower SNR region, $N U M-D$ sacrifices portion of AC_BE's throughput to achieve consistently high reliability for AC_RT. While at the higher SNR region, $N U M-D$ achieves significantly higher throughput than $N U M-S$ and also maintains AC_RT's reliability greater than $99 \%$. As a result, it successfully achieves a good balance 


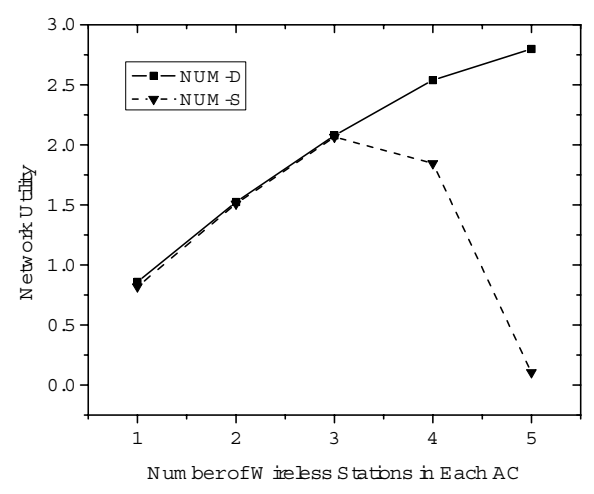

(a)

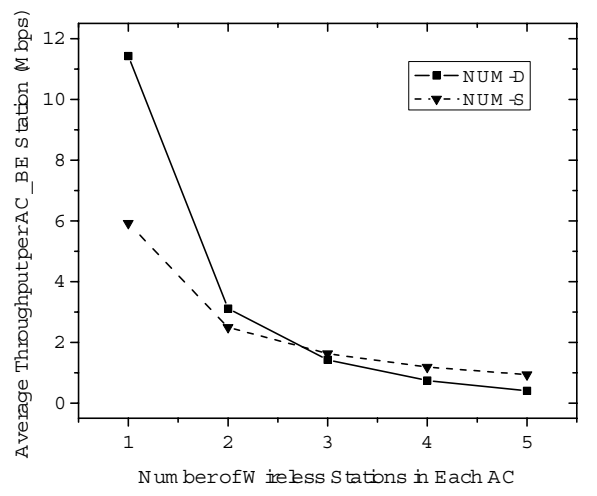

(b)

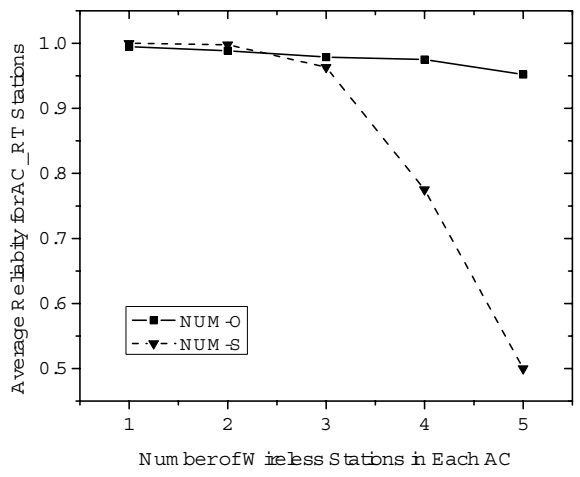

(c)

Fig. 2. Performance under different number of stations.

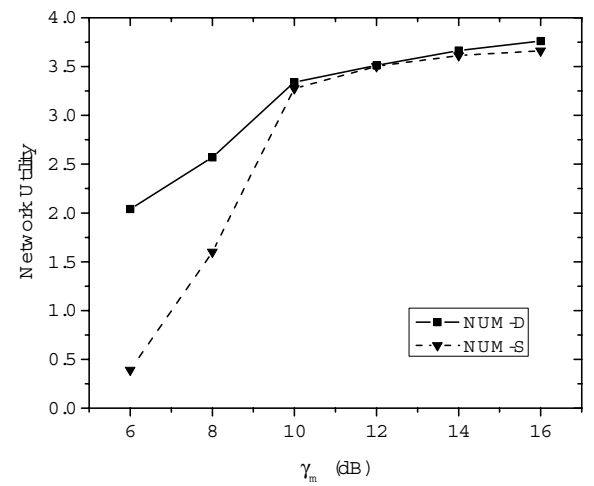

(a)

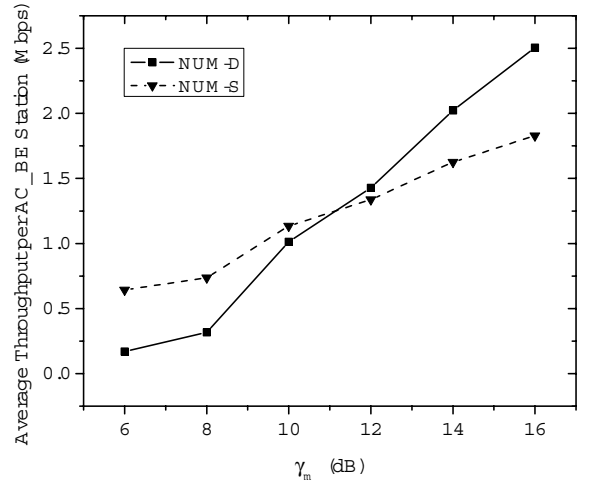

(b)

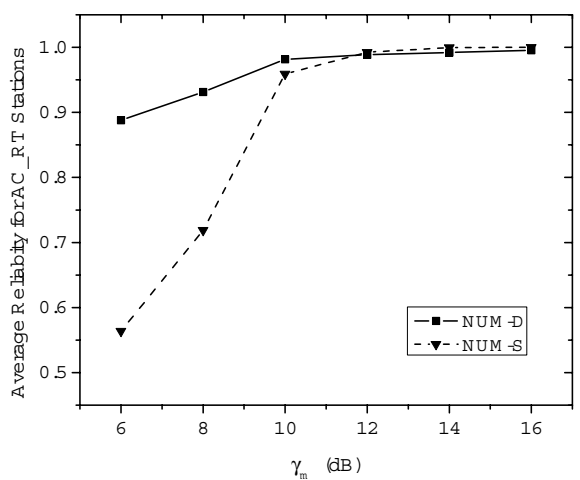

(c)

Fig. 3. Performance under different signal-to-noise ratio $\gamma_{m}$.

between throughput for best-effort traffic and reliability for real-time traffic and attains higher network utility than the $N U M-S$ scheme.

The above results show that the cross-layer network utility maximization formulation of (15) successfully balances between two access categories' QoS requirements by jointly adapting MAC and MIMO physical layer parameters.

\section{CONCLUSIONS}

This paper proposed a MAC/PHY cross-layer design for network utility maximization in a WLAN with multiple classes of traffic. The persistent probability at the MAC layer is jointly optimized with the multiplexing-diversity gain tradeoff at the physical layer with MIMO antennas. A distributed solution is achieved by variable transformation and dual-decomposition of the original problem. Numerical results show that this crosslayer design consistently outperforms a simpler solution where the MAC and PHY layers are statically designed without considering the interaction between layers. The utility-optimal cross-layer design is also shown to be flexible in adjusting the system performance in regard to tradeoff between QoS supports for different access categories of traffic. Our future work will consider extending the existing framework to a multi-hop scenario and to use a CSMA/CA MAC protocol.

\section{REFERENCES}

[1] IEEE 802.11n WG, “IEEE 802.11n Draft 2.0,” Jan. 2007.

[2] D. Tse and P. Viswanath, Fundamentals of Wireless Communication. Cambridge University Press, 2005.

[3] L. Zheng and D. N. C. Tse, "Diversity and multiplexing: A fundamental tradeoff in multiple-antenna channels," IEEE Trans. Inform. Theory, vol. 49, no. 5, pp. 1073-1096, May 2003.

[4] M. Park, S. Choi, and S. M. Nettles, "Cross-layer MAC design for wireless networks using MIMO," in Proc. of IEEE Globecom, St. Louis, MO, Nov. 2005

[5] K. Sundaresan and R. Sivakumar, "Routing in ad-hoc networks with MIMO links," in Proc. of IEEE Int'l Conf. on Network Protocols, Boston, MA, Nov. 2005.

[6] A. L. Toledo and X. Wang, "TCP performance over wireless MIMO channels with ARQ and packet combining," IEEE Trans. Mobile Comput., vol. 5, no. 3, pp. 208-223, Mar. 2006.

[7] J. Lee, M. Chiang, and A. R. Calderbank, "Price-based distributed algorithms for rate-reliability tradeoff in network utility maximization," IEEE J. Select. Areas Commun., vol. 24, no. 5, pp. 962-976, May 2006.

[8] M. Chiang, S. H. Low, A. R. Calderbank, and J. C. Doyle, "Layering as optimization decomposition: A mathematical theory of network architectures," Proceedings of the IEEE, vol. 95, no. 1, pp. 255-312, Jan. 2007.

[9] Y. Lin and V. W. S. Wong, "Adaptive tuning of MIMO-enabled 802.11e WLANs with network utility maximization," in Proc. of IEEE WCNC, Las Vegas, NV, Mar. 2008.

[10] J. Mo and J. Walrand, "Fair end-to-end window-based congestion control," IEEE/ACM Trans. Networking, vol. 8, no. 5, pp. 556-567, Oct. 2000.

[11] D. P. Bertsekas, Nonlinear Programming, 2nd ed. Athena Sci., 2004.

[12] D. Bertsekas and R. Gallager, Data networks, 2nd ed. Prentice Hall, 1992. 